\title{
RiskRank to predict systemic banking crises with common exposures
}

\author{
Peter Sarlin \\ RiskLab Finland, Arcada and \\ Hanken School of Economics, \\ Finland \\ peter@risklab.fi
}

\author{
Paolo Giudici \\ Department of Economics and \\ Management \\ University of Pavia, Italy \\ paolo.giudici@unipv.it
}

\author{
Alessandro Spelta \\ Department of Economics and \\ Finance \\ Catholic University and \\ Complexity Lab in Econ., Italy \\ alessandro.spelta@unicatt.it
}

\author{
Kaj-Mikael Björk \\ RiskLab Finland, Arcada, \\ Finland \\ kaj-mikael.bjork@arcada.fi
}

\begin{abstract}
Systemic risk has remained at the nexus of macrofinancial research and policymaking in most parts of the world. Much of the attention has focused on understanding implication of the interconnectedness of financial markets. Instead of focusing only on networks, we use and test the utility of network structures in a novel way. We use RiskRank as a framework to test the use of networks of financial systems, and particularly focus on testing the utility of the network dimension of common exposures (funding composition and portfolio overlap). RiskRank provides an ideal playground for testing the extent to which direct and common exposures perform in capturing transmission of financial crises. The results in this paper highlight the importance of common exposures. We show that funding and portfolio composition overlap are significant channels of contagion and should be accounted for when measuring systemic risk.
\end{abstract}

\section{Introduction}

The global financial crisis triggered a large number of efforts on different types of systemic risk (cf. Allen and Gale, 2000; Acharya, 2009), including macrofinancial imbalances, exogenous aggregate shocks and contagion risk. While systemic risk is oftentimes divided into cyclical and cross-sectional components (Borio, 2011), a plethora of articles have focused on the latter of the two, i.e., the interconnectedness of financial systems. This paper postulates, and tests, the utility of measuring portfolio overlap, as well as tests to what extent it helps in identifying risks at the country level.
Many authors calculate from market prices the quantiles of the estimated loss probability distribution of a bank, conditional on the occurrence of an extreme event in the financial market. Examples of articles trying to capture systemic risk from market prices include Acharya et al. (2010), Adrian and Brunnermeier (2011), Brownlees and Engle (2012), Acharya et al. (2012), Banulescu and Dumitrescu (2015) and Hautsch et al. (2014). The above studies rely on a bivariate approach, which allow calculating the risk of an institution conditional on another or on a reference market but does not address the issue of how risks are transmitted between different institutions in a multivariate framework. To address the multivariate nature of systemic risk, a large number of studies have made use of correlation network models (see, e.g., Lorenz et al. (2009); Battiston et al. (2012)). Some early studies include Billio et al. (2012) and Diebold and Yilmaz (2014), after which extensions by Barigozzi and Brownlees (2014) and Ahelegbey et al. (2016) introduce stochastic graphical models.

In contrast to the literature on correlation networks that targets the dependence structure in market prices, this paper measures correlation in network structures. As pointed out by Brunetti et al. (2015), correlations in network structures hold promise for assessing common exposures and complement direct linkages. Giudici et al. (2017) test measures of common exposures in the context of market prices, and does not put forward a generalized approach to this testing framework. This paper sheds additional light on this problem, in the context of national interbank markets. Further, this paper provides a test between direct and common exposures as a measure of risks prior to financial crises with a general purpose tool, RiskRank. A large number of studies have assessed network structures of interbank markets using the Bank of International 
Settlements (BIS) data set, including Garratt et al. (2011), Giudici and Spelta (2016), McGuire and Tarashev (2006), Minoiu and Reyes (2013).

The methodological contribution of this paper is to formally compare classical networks and correlation based networks, using appropriate predictive comparisons. Using the correlation as a measure of proximity in a multivariate framework, we provide measures of funding composition and portfolio similarities. From an applied viewpoint, we shed further light on the interpretation of country bilateral financial flows data, contained in the BIS statistics. We also combine these measures with banking crises, in a standard early-warning setup, in order to evaluate whether and to what extent they are related to the build-up of imbalances prior to crisis events.

Results suggest the predictive power of direct linkages is clearly outperformed by the other ways of defining relationships. While linkages based upon common exposures and a combination of direct and common exposures perform equally well in forecasting crisis episodes, the predictive power obtained combining the two type of network is superior for a certain parameter range. This highlights the importance of common exposures. From an economic point of view, this clearly shows that common exposures, or socalled funding/portfolio composition overlap, indeed are channels of contagion and should be accounted for when measuring systemic risk.

The paper is organized as follows. Section 2 introduces the portfolio overlap methods used and Section 3 the crisis signaling and signal evaluation methods adopted. While Section 4 describes the empirical exercise in the network structure, Section 5 presents the results of comparing our exposure measures in terms of performance in signaling a crisis through the RiskRank measure. Finally, Section 6 contains some concluding remarks and future research directions.

\section{Networks and portfolio overlap methodology}

This section presents the methods used for estimating portfolio overlap. We can represent a network by the means of a graph $G=(V ; E)$ consisting of a set $V$ of $\$ n \$$ vertices and a set $E$ of $m$ edges. A weight $w_{i, j}$, (with $i, j=1, \ldots, m$ ) can possibly be associated to each edge $(i ; j)$ and then a weighted (or valued) graph is defined.

After the recent crisis, it has been argued that network theory will enhance the understanding of financial systems, systemic risk, and the comprehension of the factors causing failures in financial markets. Usually, the financial systems are approached studying the connections among financial institutions. This is done through measuring banking liabilities and claims because credit interlinkages are inherently important for propagating, absorbing or magnifying shocks. Even if a topology of a network is known to play a major role in robustness against shocks, the lack of bilateral data have prevented the systematical investigation of the topological properties of the international financial network. Always when data are missing or confidential, correlation based networks seem to be a better alternative to classical network models. While the literature has focused on dependence structures among market prices, the focus in this paper is on correlations in network structures.

Correlation networks can be used when analyzing the structure of pairwise correlations among a set of $N$ time series. The proximity measure is defined as follows:

$$
d_{i j}=2-\sqrt{2\left(1-C_{i j}\right)}
$$

where $C_{i, j}$ is the correlation coefficient between the two time series $s_{i}$ and $s_{j}$ in a given time window. The topological analysis of the international financial network needs to be developed. In order to do so we will use the following procedure: we will associate different the time series with the different nodes of a network. Each pair of nodes can be thought to be connected by an edge, with weights that can be related to the correlation coefficient between the two corresponding time series. A proximity network of $n$ nodes can be derived by its associated $n \times n$ matrix of proximities $\boldsymbol{D}$ a weighted adjacency matrix, with elements $d_{i, j}$ described by equation 1 . Now the network is obtained and the following step is to describe a node centrality with an interconnectedness summary measure. This task is done to describe and to provide an indicator that can act as a measure of systemic importance.

In this paper, the set of nodes represent countries, while the set of edges depends on value that defines the link. The aim of the paper is to study and to compare networks of direct flows between countries' banking sectors with common exposure networks based on correlations between streams of loans. A link between two countries in a direct network represents a flow of funds, in millions of dollars, between a borrower and a lender. A link in a common exposure network, instead, measures the similarity between the funding composition or between the portfolio allocations of two countries, depending on whether in-flows or out-flows are used to compute the correlations. While in a direct network the links are directional, from a lender to a 
borrower, in common exposure networks they are undirected, and they are computed starting from the correlation between the in-flows and out-flows of a country with respect to all other countries.

\section{Crisis signaling and signal evaluation methodology}

It is possible to use standard approaches from crisis signaling with the purpose of testing to what extent direct and indirect exposures affects the overall level of stress in the banking sector. Crisis signaling and Earlywarning models are used to divide economies into precrisis and tranquil ones. This will result into a twoclass classification problem, which is a generic problem formulation in machine learning. We are aiming for a model that separates vulnerable and tranquil classes to discriminate between them by estimating the probability of being in a vulnerable state. It is also important to take into consideration the time-series dimension, when testing the predictive power of the models. Therefore, recursive real-time out-of-sample tests to assess performance will be taken. This implies that a new model is created at each quarter using the information available then. We need also to consider publication lags and the information in a realistic manner.

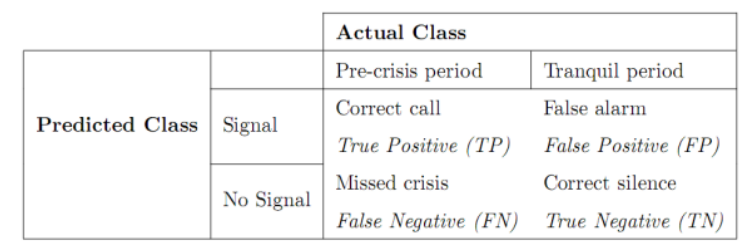

Table 1. A contingency matrix

In order to have comparable results, we will use the evaluation framework for early-warning models in (Sarlin, 2013), and mimicking an ideal leading indicator $C_{n}(h) \in\{0,1\}$ for observation $n$ (where $n=1,2, \ldots, N)$ and forecast horizon $h$, meaning that we have a binary indicator that is one during vulnerable periods and zero otherwise. For detecting events $C_{n}$, we will use a continuous measure indicating membership in a vulnerable state $p_{n} \in[0,1]$. We will then change it into a binary prediction $B_{n}$ that takes the value one if $p_{n}$ exceeds a specified threshold $\tau \in[0,1]$ and zero otherwise. The relationship between the prediction $B_{n}$ and the ideal leading indicator $C_{n}$ can then be summarized into a contingency matrix, as found in Table 1. In terms of the elements of the contingency matrix, we can differentiate between two different types of classification errors that a decision maker may be concerned with: missing crises and issuing false alarms. The next step is to define the concepts of usefulness and relative usefulness for the classification performance (as found in Sarlin, 2013). The process will define type I errors (the share of missed crises to the frequency of crises, i.e. $T_{l}=F N /(F N+T P)$ ) and type II errors (the share of issued false alarms to the frequency of tranquil periods, i.e. $\left.T_{2}=F P /(T N+F P)\right)$. The following step is to obtain the policymakers' relative preference between type I and II errors $(\mu)$ to account for the potentially imbalanced costs of errors and the unconditional probabilities of crises $P_{1}$ and tranquil periods $\mathrm{P}_{2}$ to account for the potential difference in the size of the two classes. Based on these values, we can define the loss function as:

$$
L(\mu)=\mu T_{1} P_{1}+(1-\mu) T_{2} P_{2} .
$$

The absolute usefulness of the model can be specified by comparing it to using the best guess of a policymaker (always or never signaling depending on class frequency and preferences):

$$
U_{a}(\mu)=\min \left(\mu P_{1},(1-\mu) P_{2}\right)-L(\mu) .
$$

The final step is to calculate $U_{r}$ and to compare the absolute usefulness of the model to the absolute usefulness of a model with perfect performance $L(\mu)=0$ We also strive to assess the predictive performance through calculating standard measures from the classification and machine learning literature, using the area under the receiver operating characteristic $(R O C)$ curve $(A U C)$. These measures take into consideration the preferences of a policymaker as well as more general-purpose measures to assess model performance. Other performance measures to be used in assessing the model include: (i) precision of signals $T P /(F P+T P)$; this is the share of correct signals to the frequency of signals; (ii) precision of tranquil predictions $T N /(F N+T N)$, i.e. the share of correct silence in tranquil times to the frequency of predicting tranquil time; (iii) recall of signals $T P /(F N+T P)$, i.e. the share of correct signals to the frequency of crisis times; (iv) recall of tranquil predictions $T N /(F P+T N)$, i.e. the share of correct silence in tranquil times to the frequency of tranquil times; (v) accuracy of the model $T P+T N /(F N+F P+T N+T P)$, i.e. the share of correct classifications.

\section{Network data calculations}

This section compares the use of direct exposures with common exposures in a predictive model of systemic banking crises. In short, we let vulnerability pass-through networks, where links are defined as 
direct and common exposures, and compare their predictive performance. To start with, we introduce crisis signaling as a task as well as an evaluation exercises around the task. Then, we move ahead to an empirical investigation of European crises.

For networks, the main data source in this paper is statistics from the Bank of International Settlements (BIS). The International Banking Statistics comprises consolidated banking statistics (CBS). CBS capture worldwide consolidated claims of banks headquartered in many countries, including claims of their own foreign affiliates but excluding interoffice positions. The dataset above is based upon measures used by banks from their internal risk management systems. This dataset typically include data on off-balance sheet exposures (risk transfers, guarantees and credit commitments).

We employ the consolidated banking statistics on ultimate risk basis that are based on the country where the ultimate risk or obligor resides, after taking into account risk transfers. We have also taken into consideration the fact that the statistics capture worldwide consolidated positions of the banks thus, the CBS reporting area is not synonymous of the location of the banking offices within the dataset. A reporting country should consolidate the positions of all banking entities controlled by a parent institution located in the reporting country, thus including banking entities which are actually domiciled elsewhere. With reporting institutions we mean the financial institutions whose business is to receive deposits, or close substitutes for deposits, and to grant credits or invest in securities on their own account. In this way, the set of reporting institutions should include not only commercial banks but also savings banks, credit unions or cooperative credit banks, as well as other financial credit institutions. There is a complicated aspect in the fact that a number of countries do not report their statistics on the asset side (out-flows); we have 15 fully reporting countries and more than 240 that do not report in our dataset. Another complicating factor is found in the time series with varying starting dates and of missing values. Therefore we split the analysis of the in- and out-flows in two different databases. We restrict the analysis to the 33 largest economies in the funding side (for which the received loans sum up to last 100000 billion dollars for the period from 1998 to 2013). The time period starts from the third quarter of 1998 (Q3--1998) to the last quarter of 2013 (Q4-2013). We are forced to use only 15 reporting countries on the investment side, from the third quarter of 1998 (Q3--1998) to the last quarter of 2013 (Q4-2013). The countries selected for in-flows analysis are the following: $\mathrm{AT}=$ Austria, $\mathrm{AU}=$ Australia, $\mathrm{BE}=$ Belgium, $\mathrm{BR}=$ Brazil, $\mathrm{CA}=$ Canada, $\mathrm{CH}=$
Switzerland, $\mathrm{CN}=$ Cina, $\mathrm{CZ}=$ Czech Republic, $\mathrm{DE}=$ Germany, DK = Denmark, ES = Spain, FI = Finalnd, $\mathrm{FR}=$ France, $\mathrm{GB}=$ Great Britain, $\mathrm{GR}=$ Greece, $\mathrm{HK}=$ Hong Kong, IE = Ireland, IN = India, IT = Italy, JP = Japan, KR = South Korea, KY = Cayman Islands, LU $=$ Luxemburg, $\mathrm{MX}=$ Mexico, $\mathrm{NL}=$ The Netherlands, $\mathrm{NO}=$ Norway, $\mathrm{NZ}=$ New Zeland, PL $=$ Poland, $\mathrm{PT}=$ Portugal, RU = Russia, SE = Sweden, $\mathrm{SG}=$ Singapore, US = United State. The countries selected for out-flows analysis are the following: $\mathrm{AT}=$ Austria, $\mathrm{AU}=$ Australia, $\mathrm{BE}=$ Belgium, $\mathrm{CH}=$ Switzerland, $\mathrm{DE}=$ Germany, DK = Denmark, ES = Spain, FR = France, $\mathrm{GB}=$ Great Britain, IE = Ireland, JP = Japan, NL = The Netherlands, $\mathrm{SE}=$ Sweden, $\mathrm{TW}=$ Taiwan, $\mathrm{US}=$ United States. Notice that the proposed strategy is consistent with Basel III regulation that look separately at the lending and borrowing sides of banks' balance sheet in the process of evaluating the systemic importance (Board, 2013).

\section{Results - Comparing networks with RiskRank}

In this section, we compare direct and indirect exposures with the RiskRank measure (Mezei and Sarlin, 2016). RiskRank uses two inputs: individual risk for a set of economies measured by an earlywarning model and interconnectedness across these economies. Essentially, this allows measuring the vulnerability of an individual economy, accounting for both domestic risk as well as risk stemming from exposures to other economies. In this exercise, the early-warning model follows the approach in (Holopainen and Sarlin, 2016). For an early-warning model, we need two types of data: crisis events and vulnerability indicators. The crisis events are based upon the IMF database by Icite\{laeven2013systemic\}, while the vulnerability indicators used include most common measures of widespread imbalances, such as excessive credit growth, excessive increases in stock and house prices, GDP growth, loans to deposits and debt service ratio, as well as more structural indicators, such as government debt, current account deficits and inflation. We use a standard logit model with 14 macro-financial indicators for 15 European economies and a forecast horizon of 5--12 quarters prior to crisis events, as is common in the literature. The network dimension is measured with BIS International Banking Statistics in three ways: direct, indirect (common exposures) and combined exposures. This provides ample means to compare the three types of networks.

RiskRank provides a risk measure that combines measures of individual risk and interconnectedness. In principle, it is nothing else than an aggregation 
operator for each entity $c$ that aggregates over node values (i.e., individual risk) over link values (i.e., interconnectedness). Thus, we can write RiskRank as follows:

$$
\begin{aligned}
R R_{c}\left(x_{1}, \ldots, x_{n}, x_{c}\right)= & \underbrace{x_{c}}_{\text {Individual effect of } c}+\sum_{i=1}^{n} \underbrace{\left(v\left(c_{i}\right)-\frac{1}{2} \sum_{j \neq i} I\left(c_{i}, c_{j}\right)\right) x_{i}}_{\text {Direct effect of } i \text { on } \mathrm{c}} \\
& +\underbrace{\sum_{i}^{n} \sum_{j \neq i}^{n} I\left(c_{i}, c_{j}\right) \prod\left(x_{i}, x_{j}\right)}_{\text {Indirect effect of } j \text { via } i \text { on c }}
\end{aligned}
$$

where $c$ is the evaluated node and $x_{c}$ is its associated node value. In this case, the nodes are countries and their values crisis probabilities. Further, $I\left(c_{i}, c_{j}\right)$ stands for the link between nodes $i$ and $j$ and $v\left(c_{i}\right)$ stands for the Shapley-index (average contribution of fixed element $x_{i}$ in any subset).
In the following the results of the RiskRank comparisons are given (Tables 2-9). Note that the tables reports recursive out-of-sample performance for the direct network with a forecast horizon of 5-12 quarters. The table reports in columns the following measures to assess the overall performance of the models: preferences $(\mu)$, optimal threshold $(\tau)$, absolute $\left(U_{a}\right)$ and relative $\left(U_{r}\right)$ usefulness, and $A U C=$ area under the $R O C$ curve ( $T P$ rate to $F P$ rate), $T P=$ True positives, $F P=$ False positives, $T N=$ True negatives, $F N=$ False negatives, Precision of positives $(P P)=$ $T P /(T P+F P)$, Recall of positives $(R P)=T P /(T P+F N)$, Precision of negatives $(P N)=T N /(T N+F N)$, Recall of negatives $(R N)=T N /(T N+F P)$, Accuracy $($ Acc. $)=$ $(T P+T N) /(T P+T N+F P+F N)$.

\begin{tabular}{llllllllllllll}
$\boldsymbol{\mu}$ & $\boldsymbol{\tau}$ & $\mathbf{U}_{\mathbf{a}}$ & $\mathbf{U}_{\mathbf{r}}$ & AUC & TN & TP & FN & FP & PP & RP & PN & RN & Acc. \\
\hline 0.0 & 1.00 & 0.00 & NaN & 0.92 & 747 & 0 & 76 & 1 & 0.00 & 0.00 & 0.91 & 1.00 & 0.91 \\
0.1 & 1.00 & 0.00 & -0.12 & 0.92 & 747 & 0 & 76 & 1 & 0.00 & 0.00 & 0.91 & 1.00 & 0.91 \\
0.2 & 1.00 & 0.00 & -0.05 & 0.92 & 747 & 0 & 76 & 1 & 0.00 & 0.00 & 0.91 & 1.00 & 0.91 \\
0.3 & 0.99 & 0.00 & -0.01 & 0.92 & 745 & 6 & 70 & 3 & 0.67 & 0.08 & 0.91 & 1.00 & 0.91 \\
0.4 & 0.99 & 0.00 & 0.02 & 0.92 & 745 & 6 & 70 & 3 & 0.67 & 0.08 & 0.91 & 1.00 & 0.91 \\
0.5 & 0.96 & 0.00 & 0.07 & 0.92 & 734 & 19 & 57 & 14 & 0.58 & 0.25 & 0.93 & 0.98 & 0.91 \\
0.6 & 0.96 & 0.01 & 0.13 & 0.92 & 734 & 19 & 57 & 14 & 0.58 & 0.25 & 0.93 & 0.98 & 0.91 \\
0.7 & 0.79 & 0.02 & 0.28 & 0.92 & 641 & 67 & 9 & 107 & 0.39 & 0.88 & 0.99 & 0.86 & 0.86 \\
0.8 & 0.78 & 0.04 & 0.54 & 0.92 & 635 & 69 & 7 & 113 & 0.38 & 0.91 & 0.99 & 0.85 & 0.85 \\
0.9 & 0.72 & 0.06 & 0.77 & 0.92 & 593 & 76 & 0 & 155 & 0.33 & 1.00 & 1.00 & 0.79 & 0.81 \\
1.0 & 0.00 & 0.00 & $\mathrm{NaN}$ & 0.92 & 0 & 76 & 0 & 748 & 0.09 & 1.00 & $\mathrm{NaN}$ & 0.00 & 0.09
\end{tabular}

Table 2. Predictive performance without networks (with countries selected for the in-flows analysis).

\begin{tabular}{llllllllllllll}
$\boldsymbol{\mu}$ & $\boldsymbol{\tau}$ & $\mathrm{U}_{\mathbf{a}}$ & $\mathrm{U}_{\mathbf{r}}$ & $\mathrm{AUC}$ & $\mathbf{T N}$ & $\mathbf{T P}$ & $\mathbf{F N}$ & $\mathbf{F P}$ & $\mathbf{P P}$ & $\mathbf{R P}$ & PN & RN & Acc. \\
\hline 0.0 & 0.94 & -0.02 & $\mathrm{NaN}$ & 0.94 & 732 & 34 & 42 & 16 & 0.68 & 0.45 & 0.95 & 0.98 & 0.93 \\
0.1 & 0.94 & -0.01 & -1.45 & 0.94 & 732 & 34 & 42 & 16 & 0.68 & 0.45 & 0.95 & 0.98 & 0.93 \\
0.2 & 0.94 & -0.01 & -0.39 & 0.94 & 732 & 34 & 42 & 16 & 0.68 & 0.45 & 0.95 & 0.98 & 0.93 \\
0.3 & 0.94 & 0.00 & -0.04 & 0.94 & 732 & 34 & 42 & 16 & 0.68 & 0.45 & 0.95 & 0.98 & 0.93 \\
0.4 & 0.94 & 0.00 & 0.13 & 0.94 & 732 & 34 & 42 & 16 & 0.68 & 0.45 & 0.95 & 0.98 & 0.93 \\
0.5 & 0.93 & 0.01 & 0.24 & 0.94 & 728 & 38 & 38 & 20 & 0.66 & 0.50 & 0.95 & 0.97 & 0.93 \\
0.6 & 0.91 & 0.02 & 0.33 & 0.94 & 718 & 45 & 31 & 30 & 0.60 & 0.59 & 0.96 & 0.96 & 0.93 \\
0.7 & 0.86 & 0.03 & 0.47 & 0.94 & 692 & 60 & 16 & 56 & 0.52 & 0.79 & 0.98 & 0.93 & 0.91 \\
0.8 & 0.86 & 0.04 & 0.61 & 0.94 & 692 & 60 & 16 & 56 & 0.52 & 0.79 & 0.98 & 0.93 & 0.91 \\
0.9 & 0.73 & 0.06 & 0.74 & 0.94 & 598 & 73 & 3 & 150 & 0.33 & 0.96 & 1.00 & 0.80 & 0.81 \\
1.0 & 0.00 & 0.00 & $\mathrm{NaN}$ & 0.94 & 0 & 76 & 0 & 748 & 0.09 & 1.00 & $\mathrm{NaN}$ & 0.00 & 0.09
\end{tabular}

Table 3. Predictive performance of the direct network (with countries selected for the in-flows analysis). 


\begin{tabular}{llllllllllllll}
$\boldsymbol{\mu}$ & $\boldsymbol{\tau}$ & $\mathbf{U}_{\mathbf{a}}$ & $\mathbf{U}_{\mathbf{r}}$ & $\mathbf{A U C}$ & $\mathbf{T N}$ & $\mathbf{T P}$ & $\mathbf{F N}$ & $\mathbf{F P}$ & $\mathbf{P P}$ & $\mathbf{R P}$ & $\mathbf{P N}$ & $\mathbf{R N}$ & Acc. \\
\hline 0.0 & 0.92 & -0.03 & $\mathrm{NaN}$ & 0.97 & 722 & 45 & 31 & 26 & 0.63 & 0.59 & 0.96 & 0.97 & 0.93 \\
0.1 & 0.92 & -0.02 & -2.49 & 0.97 & 722 & 45 & 31 & 26 & 0.63 & 0.59 & 0.96 & 0.97 & 0.93 \\
0.2 & 0.92 & -0.01 & -0.78 & 0.97 & 722 & 45 & 31 & 26 & 0.63 & 0.59 & 0.96 & 0.97 & 0.93 \\
0.3 & 0.92 & -0.01 & -0.21 & 0.97 & 722 & 45 & 31 & 26 & 0.63 & 0.59 & 0.96 & 0.97 & 0.93 \\
0.4 & 0.92 & 0.00 & 0.08 & 0.97 & 722 & 45 & 31 & 26 & 0.63 & 0.59 & 0.96 & 0.97 & 0.93 \\
0.5 & 0.89 & 0.01 & 0.28 & 0.97 & 713 & 56 & 20 & 35 & 0.62 & 0.74 & 0.97 & 0.95 & 0.93 \\
0.6 & 0.85 & 0.03 & 0.47 & 0.97 & 695 & 71 & 5 & 53 & 0.57 & 0.93 & 0.99 & 0.93 & 0.93 \\
0.7 & 0.84 & 0.04 & 0.65 & 0.97 & 690 & 74 & 2 & 58 & 0.56 & 0.97 & 1.00 & 0.92 & 0.93 \\
0.8 & 0.84 & 0.06 & 0.78 & 0.97 & 690 & 74 & 2 & 58 & 0.56 & 0.97 & 1.00 & 0.92 & 0.93 \\
0.9 & 0.82 & 0.07 & 0.89 & 0.97 & 675 & 76 & 0 & 73 & 0.51 & 1.00 & 1.00 & 0.90 & 0.91 \\
1.0 & 0.00 & 0.00 & $\mathrm{NaN}$ & 0.97 & 0 & 76 & 0 & 748 & 0.09 & 1.00 & $\mathrm{NaN}$ & 0.00 & 0.09
\end{tabular}

Table 4. Predictive performance of the funding composition similarity network.

\begin{tabular}{llllllllllllll}
$\boldsymbol{\mu}$ & $\boldsymbol{\tau}$ & $\mathrm{U}_{\mathbf{a}}$ & $\mathrm{U}_{\mathbf{r}}$ & $\mathbf{A U C}$ & $\mathbf{T N}$ & $\mathrm{TP}$ & $\mathbf{F N}$ & $\mathbf{F P}$ & $\mathbf{P P}$ & $\mathbf{R P}$ & $\mathbf{P N}$ & $\mathbf{R N}$ & Acc. \\
\hline 0.0 & 0.95 & -0.02 & $\mathrm{NaN}$ & 0.96 & 734 & 32 & 44 & 14 & 0.70 & 0.42 & 0.94 & 0.98 & 0.93 \\
0.1 & 0.95 & -0.01 & -1.24 & 0.96 & 734 & 32 & 44 & 14 & 0.70 & 0.42 & 0.94 & 0.98 & 0.93 \\
0.2 & 0.95 & -0.01 & -0.32 & 0.96 & 734 & 32 & 44 & 14 & 0.70 & 0.42 & 0.94 & 0.98 & 0.93 \\
0.3 & 0.95 & 0.00 & -0.01 & 0.96 & 734 & 32 & 44 & 14 & 0.70 & 0.42 & 0.94 & 0.98 & 0.93 \\
0.4 & 0.95 & 0.01 & 0.14 & 0.96 & 734 & 32 & 44 & 14 & 0.70 & 0.42 & 0.94 & 0.98 & 0.93 \\
0.5 & 0.88 & 0.01 & 0.28 & 0.96 & 709 & 60 & 16 & 39 & 0.61 & 0.79 & 0.98 & 0.95 & 0.93 \\
0.6 & 0.88 & 0.02 & 0.45 & 0.96 & 709 & 60 & 16 & 39 & 0.61 & 0.79 & 0.98 & 0.95 & 0.93 \\
0.7 & 0.85 & 0.04 & 0.58 & 0.96 & 692 & 68 & 8 & 56 & 0.55 & 0.89 & 0.99 & 0.93 & 0.92 \\
0.8 & 0.85 & 0.05 & 0.71 & 0.96 & 692 & 68 & 8 & 56 & 0.55 & 0.89 & 0.99 & 0.93 & 0.92 \\
0.9 & 0.83 & 0.07 & 0.82 & 0.96 & 677 & 70 & 6 & 71 & 0.50 & 0.92 & 0.99 & 0.91 & 0.91 \\
1.0 & 0.00 & 0.00 & $\mathrm{NaN}$ & 0.96 & 0 & 76 & 0 & 748 & 0.09 & 1.00 & $\mathrm{NaN}$ & 0.00 & 0.09
\end{tabular}

Table 5. Predictive performance of the combined network for in-flows.

\begin{tabular}{llllllllllllll}
$\boldsymbol{\mu}$ & $\boldsymbol{\tau}$ & $\mathbf{U}_{\mathbf{a}}$ & $\mathbf{U}_{\mathbf{r}}$ & AUC & TN & TP & FN & FP & PP & RP & PN & RN & Acc. \\
\hline 0.0 & 1.00 & -0.00 & - Inf & 0.93 & 527 & 0 & 52 & 1 & 0.00 & 0.00 & 0.91 & 0.99 & 0.90 \\
0.1 & 1.00 & -0.00 & -0.17 & 0.93 & 527 & 0 & 52 & 1 & 0.00 & 0.00 & 0.91 & 0.99 & 0.90 \\
0.2 & 1.00 & -0.00 & -0.07 & 0.93 & 527 & 0 & 52 & 1 & 0.00 & 0.00 & 0.91 & 0.99 & 0.90 \\
0.3 & 1.00 & -0.00 & -0.04 & 0.93 & 527 & 0 & 52 & 1 & 0.00 & 0.00 & 0.91 & 0.99 & 0.90 \\
0.4 & 0.98 & -0.00 & -0.00 & 0.93 & 523 & 7 & 45 & 5 & 0.58 & 0.13 & 0.92 & 0.99 & 0.91 \\
0.5 & 0.94 & 0.00 & 0.09 & 0.93 & 513 & 20 & 32 & 15 & 0.57 & 0.38 & 0.94 & 0.97 & 0.91 \\
0.6 & 0.92 & 0.01 & 0.19 & 0.93 & 506 & 25 & 27 & 22 & 0.53 & 0.48 & 0.94 & 0.95 & 0.91 \\
0.7 & 0.83 & 0.02 & 0.33 & 0.93 & 471 & 42 & 10 & 57 & 0.42 & 0.80 & 0.97 & 0.89 & 0.88 \\
0.8 & 0.77 & 0.03 & 0.53 & 0.93 & 443 & 49 & 3 & 85 & 0.36 & 0.94 & 0.99 & 0.83 & 0.84 \\
0.9 & 0.76 & 0.06 & 0.76 & 0.93 & 438 & 50 & 2 & 90 & 0.35 & 0.96 & 0.99 & 0.82 & 0.84 \\
1.0 & 0.00 & 0.00 & $-I n f$ & 0.93 & 0 & 52 & 0 & 528 & 0.08 & 1.00 & NaN & 0.00 & 0.08
\end{tabular}

Table 6. Predictive performance without network (with countries selected for out-flows analysis). 


\begin{tabular}{llllllllllllll}
$\boldsymbol{\mu}$ & $\boldsymbol{\tau}$ & $\mathrm{U}_{\mathbf{a}}$ & $\mathrm{U}_{\mathbf{r}}$ & AUC & TN & TP & FN & FP & PP & RP & PN & RN & Acc. \\
\hline 0.0 & 0.93 & -0.02 & - Inf & 0.96 & 514 & 30 & 22 & 14 & 0.68 & 0.57 & 0.95 & 0.97 & 0.93 \\
0.1 & 0.93 & -0.01 & -1.84 & 0.96 & 514 & 30 & 22 & 14 & 0.68 & 0.57 & 0.95 & 0.97 & 0.93 \\
0.2 & 0.93 & -0.00 & -0.50 & 0.96 & 514 & 30 & 22 & 14 & 0.68 & 0.57 & 0.95 & 0.97 & 0.93 \\
0.3 & 0.93 & -0.00 & -0.05 & 0.96 & 514 & 30 & 22 & 14 & 0.68 & 0.57 & 0.95 & 0.97 & 0.93 \\
0.4 & 0.93 & 0.00 & 0.17 & 0.96 & 514 & 30 & 22 & 14 & 0.68 & 0.57 & 0.95 & 0.97 & 0.93 \\
0.5 & 0.93 & 0.01 & 0.30 & 0.96 & 514 & 30 & 22 & 14 & 0.68 & 0.57 & 0.95 & 0.97 & 0.93 \\
0.6 & 0.87 & 0.02 & 0.43 & 0.96 & 496 & 44 & 8 & 32 & 0.57 & 0.84 & 0.98 & 0.93 & 0.93 \\
0.7 & 0.85 & 0.03 & 0.59 & 0.96 & 488 & 48 & 4 & 40 & 0.54 & 0.92 & 0.99 & 0.92 & 0.92 \\
0.8 & 0.85 & 0.05 & 0.73 & 0.96 & 488 & 48 & 4 & 40 & 0.54 & 0.92 & 0.99 & 0.92 & 0.92 \\
0.9 & 0.79 & 0.06 & 0.85 & 0.96 & 458 & 52 & 0 & 70 & 0.42 & 1.00 & 1.00 & 0.86 & 0.87 \\
1.0 & 0.00 & 0.00 & - Inf & 0.96 & 0 & 52 & 0 & 528 & 0.08 & 1.00 & NaN & 0.00 & 0.08
\end{tabular}

Table 7. Predictive performance of the direct network (with countries selected for out-flows analysis).

\begin{tabular}{llllllllllllll}
$\boldsymbol{\mu}$ & $\boldsymbol{\tau}$ & $\mathrm{U}_{\mathbf{a}}$ & $\mathrm{U}_{\mathbf{r}}$ & AUC & TN & TP & FN & FP & PP & RP & PN & RN & Acc. \\
\hline 0.0 & 0.88 & -0.04 & -Inf & 0.96 & 501 & 45 & 7 & 27 & 0.62 & 0.86 & 0.98 & 0.94 & 0.94 \\
0.1 & 0.88 & -0.03 & -3.80 & 0.96 & 501 & 45 & 7 & 27 & 0.62 & 0.86 & 0.98 & 0.94 & 0.94 \\
0.2 & 0.88 & -0.02 & -1.21 & 0.96 & 501 & 45 & 7 & 27 & 0.62 & 0.86 & 0.98 & 0.94 & 0.94 \\
0.3 & 0.88 & -0.00 & -0.34 & 0.96 & 501 & 45 & 7 & 27 & 0.62 & 0.86 & 0.98 & 0.94 & 0.94 \\
0.4 & 0.88 & 0.00 & 0.08 & 0.96 & 501 & 45 & 7 & 27 & 0.62 & 0.86 & 0.98 & 0.94 & 0.94 \\
0.5 & 0.87 & 0.01 & 0.34 & 0.96 & 499 & 47 & 5 & 29 & 0.61 & 0.90 & 0.99 & 0.94 & 0.94 \\
0.6 & 0.87 & 0.02 & 0.53 & 0.96 & 499 & 47 & 5 & 29 & 0.61 & 0.90 & 0.99 & 0.94 & 0.94 \\
0.7 & 0.85 & 0.04 & 0.67 & 0.96 & 491 & 51 & 1 & 37 & 0.57 & 0.98 & 0.99 & 0.94 & 0.94 \\
0.8 & 0.84 & 0.05 & 0.80 & 0.96 & 487 & 52 & 0 & 41 & 0.55 & 1.00 & 1.00 & 0.92 & 0.92 \\
0.9 & 0.84 & 0.07 & 0.91 & 0.96 & 487 & 52 & 0 & 41 & 0.55 & 1.00 & 1.00 & 0.92 & 0.92 \\
1.0 & 0.00 & 0.00 & - Inf & 0.96 & 0 & 52 & 0 & 528 & 0.08 & 1.00 & NaN & 0.00 & 0.08
\end{tabular}

Table 8. Predictive performance of the portfolio similarity network. 


\begin{tabular}{llllllllllllll}
$\boldsymbol{\mu}$ & $\boldsymbol{\tau}$ & $\mathbf{U}_{\mathbf{a}}$ & $\mathrm{U}_{\mathbf{r}}$ & AUC & TN & TP & FN & FP & PP & RP & PN & RN & Acc. \\
\hline 0.0 & 0.92 & -0.03 & - Inf & 0.96 & 509 & 33 & 19 & 19 & 0.63 & 0.63 & 0.96 & 0.96 & 0.93 \\
0.1 & 0.92 & -0.02 & -2.65 & 0.96 & 509 & 33 & 19 & 19 & 0.63 & 0.63 & 0.96 & 0.96 & 0.93 \\
0.2 & 0.92 & -0.01 & -0.82 & 0.96 & 509 & 33 & 19 & 19 & 0.63 & 0.63 & 0.96 & 0.96 & 0.93 \\
0.3 & 0.92 & -0.00 & -0.21 & 0.96 & 509 & 33 & 19 & 19 & 0.63 & 0.63 & 0.96 & 0.96 & 0.93 \\
0.4 & 0.92 & 0.00 & 0.08 & 0.96 & 509 & 33 & 19 & 19 & 0.63 & 0.63 & 0.96 & 0.96 & 0.93 \\
0.5 & 0.92 & 0.01 & 0.26 & 0.96 & 509 & 33 & 19 & 19 & 0.63 & 0.63 & 0.96 & 0.96 & 0.93 \\
0.6 & 0.87 & 0.02 & 0.40 & 0.96 & 495 & 43 & 9 & 33 & 0.56 & 0.82 & 0.98 & 0.93 & 0.92 \\
0.7 & 0.84 & 0.03 & 0.57 & 0.96 & 484 & 49 & 3 & 44 & 0.52 & 0.94 & 0.99 & 0.91 & 0.91 \\
0.8 & 0.84 & 0.05 & 0.73 & 0.96 & 484 & 49 & 3 & 44 & 0.52 & 0.94 & 0.99 & 0.91 & 0.91 \\
0.9 & 0.84 & 0.06 & 0.84 & 0.96 & 484 & 49 & 3 & 44 & 0.52 & 0.94 & 0.99 & 0.91 & 0.91 \\
1.0 & 0.00 & 0.00 & - Inf & 0.96 & 0 & 52 & 0 & 528 & 0.08 & 1.00 & NaN & 0.00 & 0.08
\end{tabular}

Table 9. Predictive performance of the combined network for out-flows.

\section{Conclusion and future work}

Measuring portfolio similarity is a central task when modeling systemic risk and interconnectedness in financial systems, particularly for complementing measures based upon direct exposures. Systemic risk, in itself, concerns the risks posed by balance sheet relationships and interdependencies among players in a system or market, where the failure of a single entity can cause a cascading failure, which could potentially bring down an entire system or market. These balance sheet linkages can be represented by a network that describes the mutual relationships between the different economical agents involved. In this paper, we have focused on measuring portfolio overlap, and assessing the utility of common exposures as a channel for transmission effects. In our contribution we have also shown that correlation network models that aim at capturing the multivariate network structure provide suitable means for representing the indirect dimension of systemic risk through common exposures. We have combined our proposed measures with banking crises data, in order to assess whether and to what extent they are related to the build-up of imbalances prior to crisis events. The exercise clearly shows that common exposures are an important channel of contagion and should be accounted for when measuring systemic risk.

Future work should cover general development of the RiskRank approach to also incorporate dynamic iterations and feedback effects. For the specific purpose in this paper, one could also think of various other types of network linkages that could be used for measuring the efficacy of transmission channels.

\section{References}

Acharya, V., Engle, R., and Richardson, M. (2012). Capital shortfall: A new approach to ranking and regulating systemic risks. The American Economic Review, 102(3):59-64.

Acharya, V. V. (2009). A theory of systemic risk and design of prudential bank regulation. Journal of Financial stability, 5(3):224-255.

Acharya, V. V., Pedersen, L. H., Philippon, T., and Richardson, M. P. (2010). Measuring systemic risk.

Adrian, T. and Brunnermeier, M. K. (2011). Covar. Technical report, National Bureau of Economic Research.

Ahelegbey, D. F., Billio, M., and Casarin, R. (2016). Bayesian graphical models for structural vector autoregressive processes. Journal of Applied Econometrics, $31(2)$.

Allen, F. and Gale, D. (2000). Financial contagion. Journal of political economy, 108(1):1-33.

Barigozzi, M. and Brownlees, C. T. (2014). Nets: Network estimation for time series. Available at SSRN 2249909.

Banulescu, G.-D. and Dumitrescu, E.-I. (2015). Which are the sifis? a component expected shortfall approach to systemic risk. Journal of Banking \& Finance, 50:575-588.

Battiston, S., Gatti, D. D., Gallegati, M., Greenwald, B., and Stiglitz, J. E. (2012). Liaisons dangereuses: Increasing connectivity, risk sharing, and systemic risk. Journal of Economic Dynamics and Control, 36(8):1121-1141.

Billio, M., Getmansky, M., Lo, A. W., and Pelizzon, L. (2012). Econometric measures of connectedness and 
systemic risk in the finance and insurance sectors. Journal of Financial Economics, 104(3):535-559.

Board, F. S. (2013). Global systemically important banksupdated assessment methodology and the higher loss absorbency requirement. Revised version july 2013.

Borio C., 2011. Implementing a macroprudential framework: Blending boldness and realism. Capitalism andSociety, 6(1).

Brownlees, C. T. and Engle, R. F. (2012). Volatility, correlation and tails for systemic risk measurement $[\mathrm{j} / \mathrm{ol}]$.

Brunetti, C., Harris, J. H., Mankad, S., and Michailidis, G. (2015). Interconnectedness in the interbank market.

Diebold, F. X. and Yilmaz, K. (2014). On the network topology of variance decompositions: Measuring the connectedness of financial firms. Journal of Econometrics, 182(1):119-134.

Garratt, R., Mahadeva, L., and Svirydzenka, K. (2011). Mapping systemic risk in the international banking network.

Giudici P., Sarlin P., Spelta A. 2017. The interconnected nature of financial systems: Direct and common exposures. Journal of Banking \& Finance. In press, https://doi.org/10.1016/j.jbankfin.2017.05.010
Hautsch, N., Schaumburg, J., and Schienle, M. (2014). Financial network systemic risk contributions. Review of Finance, page rfu010.

Giudici, P. and Spelta, A. (2016). Graphical network models for international financial flows. Journal of Business \& Economic Statistics, 34(1):128-138.

Holopainen, M. and Sarlin, P. (2016). Toward robust earlywarning models: A horse race, ensembles and model uncertainty. ECB WP, Frankfurt.

Lorenz, J., Battiston, S., and Schweitzer, F. (2009). Systemic risk in a unifying framework for cascading processes on networks. The European Physical Journal B, 71(4):441-460.

McGuire, P. and Tarashev, N. A. (2006). Tracking international bank flows. BIS Quarterly Review, December.

Mezei, J. and Sarlin, P. (2016). Riskrank: Measuring interconnected risk. Economic Modelling. DOI: 10.1016/j.econmod.2017.04.016

Minoiu, C. and Reyes, J. A. (2013). A network analysis of global banking: 1978-2010. Journal of Financial Stability, 9(2):168-184.

Sarlin, P. (2013). On policymakers loss functions and the evaluation of early warning systems.

Economics Letters, 119(1):1-7. 